Wien.Klin.Wochenschr.Educ2016·11:63 DOI 10.1007/s11812-016-0075-z

Online publiziert: 15. März 2016

(c) Springer-Verlag Wien 2016

CrossMark
Maria Klara Frey · Roela Sadushi-Kolici • Irene Lang

Klinische Abteilung für Kardiologie, Universitätsklinik für Innere Medizin II,

Medizinische Universität Wien, Wien, Österreich

\section{Erratum zu: Pulmonale Hypertension}

\section{Erratum zu:}

Wien. Klin. Wochenschr. Educ (2015)

10:73-90

DOI 10.1007/s11812-015-0074-5

Leider befindet sich im Artikel eine falsche Antwortmöglichkeit zur 6. DFP-Frage. Korrekt muss diese Frage wie folgt lauten:

? Welche Aussage bezüglich der Vasoreagibilitätsprüfung ist falsch?

$\square$ Ein positiver Responder-Status ist definiert durch einen Abfall des mPAP um mindestens $10 \mathrm{mmHg}$, auf unter 40 $\mathrm{mmHg}$.

Calcium Antagonisten sind die Therapie der Wahl bei positivem Vasoreagibilitätstest.

$\square$ Die Vasoreagibilitätsprüfung sollte nur mit Substanzen mit kurzer Halbwertzeit durchgeführt werden.

$\square$ Eine Vasoreagibilitätsprüfung sollte bei allen neudiagnostizierten Fällen von PH durchgeführt werden.

$\square$ Um den Therapieverlauf zu beurteilen, müssen Vasoreagibilitätstests regelmäßig durchgeführt werden.

\section{Korrespondenzadresse}

\section{Prof. Dr. I. Lang}

Klinische Abteilung für Kardiologie, Universitätsklinik für Innere Medizin II, Medizinische Universität Wien Währinger Gürtel 18-20, 1090 Wien irene.lang@meduniwien.ac.at 